\section{Prevalência e fatores associados ao baixo nível de atividade física entre estudantes universitários de uma universidade pública da região Nordeste - Brasil}

\section{Prevalence and factors related to low level physical activity among university students in a public university in the northeast region of Brazil}

\section{Ana Cláudia Dias Fontes ${ }^{1}$}

\section{Rodrigo Pinheiro Toledo Vianna ${ }^{2}$}

${ }^{1}$ Centro de Ciências da Saúde da Universidade Federal da Paraíba.

${ }^{2}$ Departamento de Nutrição do Centro de Ciências da Saúde da Universidade Federal da Paraíba.

Correspondência: Rodrigo Pinheiro de Toledo Vianna. Departamento de Nutrição - CCS - UFPB Campus Universitário I - Cep: 58. 090-970 - João Pessoa - PB, Brasil. Telefone: (83) 3216-7499. E-mail: vianna@ccs.ufpb.br

\section{Resumo}

Os baixos níveis de atividade física estão relacionados com o aumento da ocorrência de doenças crônicas e a redução da qualidade de vida da população. Este estudo buscou estimar a prevalência de baixo nível de atividade física entre estudantes de graduação da Universidade Federal da Paraíba, bem como encontrar fatores associados a esta condição. Trata-se de um estudo seccional estratificado por Centro, com partilha proporcional em função do ano de ingresso, do curso e do turno de estudo. A coleta de dados ocorreu no período no primeiro semestre de 2007, com a participação de 1.503 estudantes. Foram investigadas questões relativas a dados pessoais, caracterização socioeconômica, estilo de vida e saúde, avaliação nutricional e atividade física. Para mensurar nível de atividade física utilizou-se a versão longa IPAQ. A prevalência observada de baixo nível de atividade física foi de $31,2 \%$. Os estudantes com maior tempo de ingresso na universidade, os que estudam no período noturno e aqueles que passam menos tempo na universidade tiveram maior prevalência de baixo nível de atividade física. Também maior renda e classe social mostraram-se associados a maior prevalência de baixa atividade física. Considerando os riscos do baixo nível de atividade física para saúde e a importância da fase universitária como uma transição da etapa adolescente para a vida adulta, faz-se necessário o incentivo à prática de atividades físicas na universidade como medida preventiva para doenças crônicas não transmissíveis e para a melhoria da qualidade de vida na fase adulta e na velhice.

Palavras-chave: Atividade física. Epidemiologia. Saúde do jovem. 


\section{Abstract}

Low levels of physical activity are related to an increase in chronic diseases and a decrease in the population's quality of life. This study aimed to estimate insufficient physical activity among undergraduate students of Universidade Federal da Paraíba (Federal University of Paraíba), and to find factors associated with such condition. A center-stratified cross-sectional sampling study was carried out, using a proportionate share as a function of the year they started the program, type of program chosen, and time of the day they attended classes. Data were collected along the first semester of 2007 and 1,503 students were enrolled in the study. Issues concerning personal data, socioeconomic condition, lifestyle and health, nutritional assessment, and physical activity were investigated. The long version IPAQ was used to measure the level of physical activity. The prevalence observed for low level physical activity was $31.2 \%$. Students who entered university much earlier, those who attended classes in the evening, and students who spent less time at the university showed the highest prevalence of low level physical activity. Also, family income and social class were associated with a high prevalence of low level physical activity. Taking into account the risks to health resulting from insufficient physical activity and the significance of the college years as a transition from adolescence to adulthood, physical activities at the university should be encouraged as a preventive measure against non-transmissible chronic diseases and to improve quality of life along adult and senior life.

Keywords: Physical activity. Epidemiology. Youth health.

\section{Introdução}

A prática regular da atividade física é concebida como um componente fundamental para o desenvolvimento de aspectos positivos relacionados à saúde. Em vista disto, pesquisadores de diversas partes do mundo têm demonstrado preocupação com o nível de atividade física das pessoas ${ }^{1-6}$.

Ter baixo nível de atividade física é uma condição que está associada ao desenvolvimento de doenças, tais como: diabetes, infarto do miocárdio, doença arterial coronariana, câncer e obesidade. É também um dos fatores que contribui para elevar as taxas de mortalidade, aumentar os riscos de hospitalizações e de problemas psicossociais, resultando em custos elevados para a saúde pública de modo geral ${ }^{3,7,8}$.

Apesar de se conhecer os prejuízos, tanto individuais como coletivos, advindos da condição de baixo nível de atividade física, observa-se uma tendência nas ultimas décadas de redução dos níveis de atividade física das populações. Tal fenômeno tem se constituído em importante campo de investigação, com destaque para os níveis de atividade física de adolescentes e, em especial, jovens universitários por dois motivos: porque o ingresso na universidade é um evento marcado por novas relações sociais, com a possibilidade de adoção de estilo de vida sedentário; e porque a juventude é uma fase bastante oportuna para se colocar em prática medidas preventivas. É nesta fase que o estilo de vida está sendo consolidado juntamente com a independência do jovem ${ }^{5,9-12}$.

No Brasil não se dispõe de uma base de dados sólida a respeito da prevalência de baixo nível de atividade física da população ${ }^{13}$ e são especialmente escassos os estudos sobre o perfil da atividade física de estudantes universitários. Assim, essa pesquisa teve como objetivo identificar a prevalência de baixo nível de atividade física entre os estudantes universitários da Universidade Federal da Paraíba e os fatores a ela relacionados. Estas informações serão necessárias para subsidiar comparações 
nacionais e internacionais, e dar suporte para a construção de indicadores de saúde para o planejamento de ações e formulações de políticas.

\section{Metodologia}

Realizou-se um estudo seccional com a população de estudantes universitários de graduação da Universidade Federal da Paraíba - UFPB, Campus I, localizado no município de João Pessoa-PB, no primeiro semestre de 2007.

Foi realizada técnica de amostragem estratificada com correção para populações finitas, onde cada Centro foi considerado um estrato. Para o cálculo do tamanho da amostra acatou-se o valor antecipado do evento estudado, alunos com baixo nível de atividade física, igual a $50 \%(\mathrm{P}=0,50)$. Isso determina maior aproximação para o valor da variância da característica da população, o que é traduzido pelo produto de (P Q), sendo Q a medida complementar da probabilidade $\mathrm{P}$, ou (1-P), determinandose desse modo maior tamanho da amostra para a precisão fixada. Neste caso optou-se por uma precisão de 5 pontos percentuais, que significa o valor máximo permitido para a diferença entre um resultado da amostra e os valores reais das frequências estudadas. Finalmente utilizou-se um nível de confiança de $90 \%$. Com estes estimadores, o número de indivíduos necessários para compor a amostra foi de 1.442 estudantes universitários $^{14,15}$

Para garantir a representatividade da amostra em cada Centro foi feita a distribuição dos alunos a serem entrevistados considerando o curso, o ano de ingresso e o turno de estudo, adotando-se o critério de partilha proporcional. Os alunos foram recrutados nos locais de maior fluxo em horários livres, tais como as praças e centros de convivência, até completar a distribuição esperada do plano amostral pré-definido. Para verificar a ocorrência de viés de seleção foi construída uma tabela de contingência com os valores calculados de amostra para cada centro e o total de entrevistas realiza- das. Utilizou-se o teste qui-quadrado com nível de significância de $5 \%$ para verificar se houve diferença entre a distribuição calculada e aquela realizada em cada Centro.

Foram elegíveis para participar do estudo todos os discentes de graduação ingressados entre os anos de 2003-2006. Discentes com problemas de saúde que impossibilitam a realização de atividades físicas foram excluídos.

O estudo foi aprovado pelo Comitê de Ética em Pesquisa do Centro de Ciências da Saúde da Universidade Federal da Paraíba e todos participantes assinaram o termo de consentimento livre e esclarecido.

O protocolo de pesquisa foi composto com um questionário de autopreenchimento contendo perguntas sobre dados pessoais, caracterização socioeconômica, estilo de vida e saúde e atividade física.

Os dados pessoais investigados foram sexo, idade, estado civil, se o aluno tem filhos, com quem mora e se realiza trabalho remunerado além da universidade.

Para a caracterização socioeconômica, além da renda familiar foi utilizada metodologia da ANEP, que distribui as pessoas em classes de acordo com o escore total obtido na somatória de uma lista pré-definida de bens de cada participante ${ }^{16}$. Sobre o estilo de vida foi investigada a frequência do consumo de bebida alcoólica, tabagismo e a autopercepção do estado de saúde, da qualidade do sono e se faz ou não dieta.

A avaliação da atividade física foi realizada utilizando-se o Questionário Internacional de Atividade Física-IPAQ (versão longa). Foram calculados os gastos de energia em cada domínio de atividade (trabalho, transporte, atividades domésticas, lazer e total), expressos em MET (measure energy total) para a computação do nível de atividade física ${ }^{17}$.

Segundo o seu nível de atividade física, os graduandos foram classificados em alto, moderado e baixo. Considerou-se como alto nível aqueles que realizavam atividade vigorosa três ou mais dias por semana, atingindo pelo menos 1.500 MET-minutos/ semana, ou sete ou mais dias de qualquer 
combinação de caminhada e atividade física vigorosa ou moderada, atingindo 3.000 MET-minutos/semana. Como nível moderado foram classificados todos os que alcançaram três ou mais dias de atividade vigorosa por pelo menos 20minutos/dia, ou cinco ou mais dias de atividade moderada e/ ou caminhada por 30 minutos/dia, ou ainda cinco ou mais dias de alguma combinação de caminhada, atividade moderada ou vigorosa atingindo no mínimo 600 METminutos/semana. Finalmente, como baixo nível de atividade física foram considerados aqueles que não alcançaram nenhum dos critérios das categorias anteriores ${ }^{17}$.

Além do questionário de autopreenchimento, antropometristas treinados segundo as recomendações de Guedes e Guedes $^{18}$ avaliaram o estado nutricional dos graduandos pelo indicador de Índice de Massa Corporal (IMC), calculado a partir das medidas de massa e estatura. Os equipamentos utilizados foram estadiômetro, modelo Seca, com escala de precisão de $0,1 \mathrm{~cm}$, e balança digital com precisão de $100 \mathrm{~g}$ e capacidade de $150 \mathrm{~kg}$. De acordo com o Consenso Latino-Americano de Obesida$\mathrm{de}^{19}$, sobrepeso foi considerado para IMC entre $25-29,9 \mathrm{~kg} / \mathrm{m}^{2}$ e obesidade para IMC a partir de $30 \mathrm{~kg} / \mathrm{m}^{2}$.

Todos os dados foram digitados no programa Microsoft Office Access, com máscara contendo filtros para minimizar os erros de digitação e depois exportados para o programa estatístico Statistical Package for Science Social, versão 15.0.

Foi realizada análise exploratória dos dados para verificação de inconsistências, que, quando encontradas, foram corrigidas, voltando ao questionário original. Quando isso não pôde ser feito, o dado foi considerado como perdido.

Foi feita análise descritiva das variáveis do estudo e os resultados expressos em tabelas de frequência e gráficos. Posteriormente foram realizados testes de associação entre a variável dependente, baixo nível de atividade física, categorizada como dicotômica (sim ou não), e as demais variáveis do estudo, utilizando-se o teste qui-quadrado com nível de significância de $10 \%$ para a rejeição da hipótese de não associação. Este nível de significância foi adotado para diminuir a probabilidade de ocorrência do erro tipo II, aceitar a hipótese de não associação quando na verdade ela é verdadeira, mesmo que isto implique em aumentar a probabilidade de ocorrência do erro tipo I, rejeitar indevidamente a hipótese nula. Em termos práticos, este critério de nível de significância aumenta a sensibilidade do estudo para encontrar associações entre as características relacionadas.

As variáveis que apresentaram associação estatística no teste qui-quadrado foram selecionadas para a construção de um modelo de regressão logística multivariada, com baixo nível de atividade física definido como variável dependente. Realizou-se a inclusão progressiva de cada variável baseado na estatística Wald, que fornece a significância estatística para cada coeficiente estimado, equivalente ao valor $t$ utilizado para avaliar a significância de cada coeficiente na regressão múltipla. Os pontos de corte utilizados para cada passo foram probabilidade igual ou menor que $10 \%$ para a variável entrar no modelo, e 15\% para ser excluída, possibilitando que a variável permanecesse no modelo mesmo que diminuísse sua significância estatística até o limite de $15 \%$.

O tempo gasto sentado durante um dia de semana e um dia do fim de semana típico foi comparado por seus valores medianos.

\section{Resultados}

Participaram da pesquisa 1.503 estudantes, representando a população dos seis Centros de Ensino do Campus I da UFPB. Não houve diferença entre o número de alunos planejados na amostragem e o número de alunos selecionados, garantindo a representatividade da amostra (Tabela 1 ).

A média de idade observada foi de 22,3 anos (IC 95\%: 22,1 - 22,5 anos), sendo uma pequena maioria do sexo feminino $(55,9 \%)$. Maior quantitativo de alunos morava com a família $(75,1 \%)$, eram solteiros $(90,6 \%)$, não tinham filhos $(92,6 \%)$, não realizavam 
Tabela 1 - Distribuição dos alunos segundo o plano amostral e o número de entrevistas realizadas por Centro. UFPB, 2007.

Table 1 - Students according to sample size and interviews per Center. UFPB, 2007.

\begin{tabular}{lcccc}
\hline Centro* $^{*}$ & $\begin{array}{c}\text { Total de } \\
\text { Alunos }\end{array}$ & $\begin{array}{c}\text { Amostra } \\
\text { calculada }\end{array}$ & $\begin{array}{c}\text { Entrevistas } \\
\text { realizadas }\end{array}$ & $\begin{array}{c}\text { Porcentagem do } \\
\text { esperado (\%) }\end{array}$ \\
\hline CCEN & 2010 & 239 & 243 & 101,7 \\
CCSA & 2670 & 246 & 275 & 111,8 \\
CCHLA & 4177 & 255 & 277 & 108,6 \\
CCS & 2810 & 247 & 228 & 92,3 \\
CT & 1811 & 236 & 258 & 109,3 \\
CE & 1145 & 219 & 222 & 101,4 \\
Total & 14623 & 1442 & 1503 & 104,1 \\
\hline
\end{tabular}

* CCEN: Centro de Ciências Exatas e da Natureza, CCSA: Centro de Ciências Sociais Aplicadas, CCHLA: Centro de Ciências Humanas, Letras e Artes, CCS: Centro de Ciências da Saúde, CT:Centro de Tecnologia e CE:Centro de Educação.

Qui-quadrado $=3,05$, g.l. $=5, p=0,69$ Chi-square $=3.05$, g.l. $=5, p=0.69$

trabalho remunerado além da universidade $(60,5 \%)$ e tinham suas despesas custeadas pelos pais $(57,7 \%)$.

A frequência de discentes que referiram ter renda familiar acima de cinco salários mínimos foi de $39,7 \%$, enquanto, segundo a classificação da ANEP, 63,2\% se enquadravam nas Classes "A" e "B".

A prevalência observada de alunos com baixo nível de atividade física foi de $31,2 \%$. A maior prevalência de baixo nível de atividade física foi observada no Centro de Ciências Sociais Aplicadas - CCSA (45,5\%), e as menores no Centro de Ciências da Saúde - CCS (19,7\%) e no Centro de Ciências
Humanas Letras e Artes- CCHLA (25,3\%). Os demais Centros apresentaram prevalências semelhantes à proporção total. Também no total, os alunos matriculados nos cursos noturnos apresentaram maior prevalência de baixo nível de atividade física, quando comparados com aqueles do período diurno (41,0\% x 27,1\%, p<0,0005).

Os alunos com maior tempo de ingresso na universidade tiveram uma tendência de diminuição da intensidade da atividade física realizada, reduzindo inicialmente a frequência de atividade alta para moderada e depois de moderada para baixa $(\mathrm{p}=0,013)$, como pode ser observado na Figura 1.

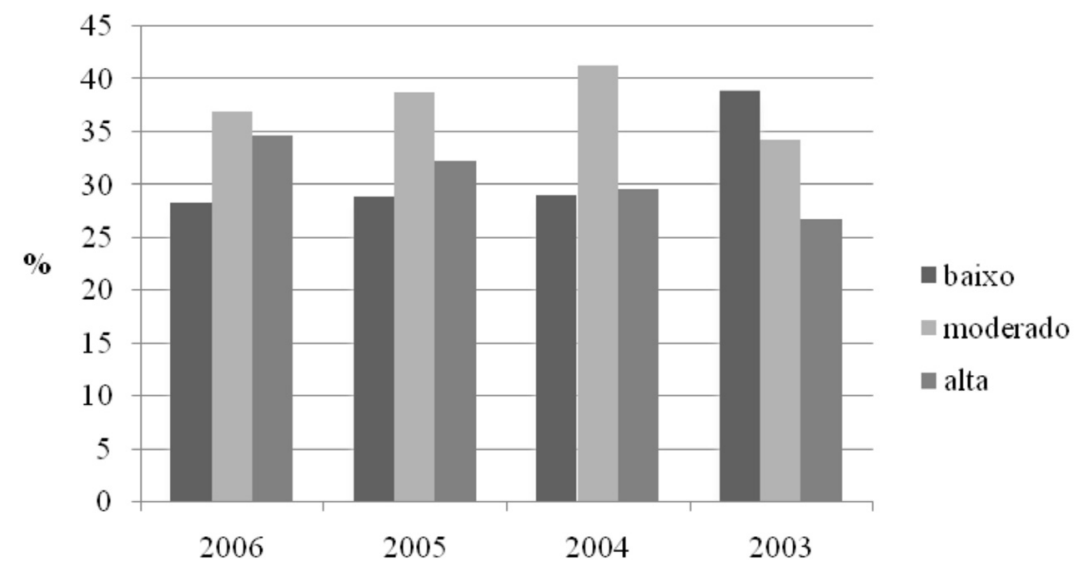

Ano de Ingresso

Figura 1 - Frequência dos diferentes níveis de atividade física realizada pelos estudantes em função do ano de ingresso ( $n=1.503)$. UFPB, 2007.

Figure 1 - Levels of physical activity by student year of enrollment in University. UFPB, 2007. 
Quando observadas as características de estilo de vida dos estudantes, $9,1 \%$ referiram fumar esporadicamente e $4,3 \%$ diariamente. Referiram que nunca consomem bebida alcoólica $39,5 \%$ dos estudantes e 19,8\% reconheceram consumir com frequência semanal ou diária.

A auto-avaliação do estado de saúde de cada discente foi descrito como excelente ou bom por $74,9 \%$ da amostra, e apenas $2 \%$ dos estudantes se classificaram como tendo estado de saúde ruim. Da mesma forma, 60,6\% referiram dormir bem sempre ou quase sempre; $10,4 \%$ relataram não ter boa qualidade de sono. Estar em dieta foi referido por $10,1 \%$ da amostra. Com relação à avaliação do estado nutricional, $21,0 \%$ dos estudantes apresentaram excesso de peso, sendo 15,3\% de sobrepeso e $5,7 \%$ de obesidade.

De acordo com o teste qui-quadrado, as variáveis que tiveram associação estatística com o baixo nível de atividade física foram: o turno de estudo, ano de ingresso na universidade, tempo diário de permanência na universidade, com quem mora, se realiza ou não trabalho remunerado, forma de custeio das próprias despesas, a renda familiar, a classificação socioeconômica segundo $\mathrm{ANEP}^{16}$, a autopercepção de saúde, da qualidade do sono e se está fazendo, ou não, algum tipo de dieta.

As variáveis sexo, idade, estado civil, ter ou não filhos, consumo de fumo e de álcool e a avaliação antropométrica não tiveram associação com o baixo nível de atividade física.

O resultado do modelo de regressão logística multivariada, com a inclusão progressiva de todas as variáveis com associação identificada na análise bivariada, controlado por idade e sexo, está mostrado na Tabela 2.

Realizar ou não trabalho remunerado, a forma de custeio das despesas e a autoper-

Tabela 2 - Fatores associados ao baixo nível de atividade física ( $\mathrm{n}=1.503)$. UFPB, 2007.

Table 2 - Factors associated with low level physical activity ( $n=1,503)$. UFPB, 2007.

\begin{tabular}{|c|c|c|c|}
\hline Variáveis & & $\mathrm{OR}^{*}$ & IC (90\%) \\
\hline \multirow[t]{2}{*}{ Turno de estudo } & (noturno) & 1,86 & $1,46-2,38$ \\
\hline & (diurno) & 1,00 & - \\
\hline \multirow[t]{4}{*}{ Ano de ingresso } & $(2003)$ & 1,73 & $1,28-2,34$ \\
\hline & (2004) & 1,16 & $0,85-1,58$ \\
\hline & (2005) & 1,07 & $0,81-1,41$ \\
\hline & $(2006)$ & 1,00 & - \\
\hline \multirow[t]{3}{*}{ Tempo diário na universidade } & (até 4h) & 1,61 & $1,18-2,19$ \\
\hline & $(5-8 h)$ & 1,50 & $1,16-1,94$ \\
\hline & (mais de 9 horas) & 1,00 & - \\
\hline \multirow[t]{3}{*}{ Com quem mora } & (família) & 2,38 & $1,41-4,01$ \\
\hline & (com amigos) & 1,51 & $0,82-2,78$ \\
\hline & (sozinho) & 1,00 & - \\
\hline \multirow[t]{3}{*}{ Renda familiar } & (5 -10 SM) & 1,62 & $1,23-2,13$ \\
\hline & $(3-5 \mathrm{SM})$ & 1,24 & $0,94-1,62$ \\
\hline & $(0-3 \mathrm{SM})$ & 1,00 & - \\
\hline \multirow[t]{4}{*}{ Classe social } & $\left(“ A^{\prime \prime}\right)$ & 3,12 & $1,56-6,24$ \\
\hline & $(" B ")$ & 2,16 & $1,14-4,10$ \\
\hline & $\left(“ C^{\prime \prime}\right)$ & 2,28 & $1,22-4,28$ \\
\hline & $(“ D / E ")$ & 1,00 & \\
\hline \multirow[t]{2}{*}{ Faz dieta } & (não) & 1,63 & $1,20-2,37$ \\
\hline & $(\operatorname{sim})$ & 1,00 & \\
\hline
\end{tabular}

*Valores de Odds Ratio controlados para idade e sexo.

* Odds Ratio controlled by age and gender. 
cepção do estado de saúde e da qualidade do sono perderam significância estatística e não foram incluídos no modelo.

De acordo com a regressão logística, têm maior chance de ter um comportamento com baixo nível de atividade física os alunos do curso noturno (1,86 vezes mais quando comparados com os do diurno), os ingressos há quatro anos (1,73 vezes quando comparados com os alunos do primeiro ano) e aqueles que passam menos tempo por dia na universidade. Com relação ao nível socioeconômico, morar com a família (2,38 vezes) e pertencer às classes sociais mais elevadas, confirmado pela variável de renda familiar (1,62 vezes) e da classificação econômica da $\operatorname{ANEP}^{16}$ ( 3,12 vezes), também aumentam as chances de ter um comportamento com baixa atividade física. Finalmente, não estar realizando dieta alimentar também aumenta a chance de baixo nível de atividade física (1,63 vezes).

A mediana do tempo gasto sentado em um dia de semana típico foi de oito horas, enquanto que em um dia de final de semana típico foi de seis horas. Utilizando a mediana para a comparação dos grupos com diferentes níveis de atividade física, a única diferença observada foi entre aqueles com alta atividade física que apresentaram tempo mediano sentado em um dia de semana típico de sete horas, e portanto inferior aos classificados com atividade moderada e com baixa atividade física.

\section{Discussão}

A pesquisa realizada avaliou a atividade física e fatores associados ao baixo nível de atividade física dos estudantes de uma Universidade federal da região nordeste. $\mathrm{O}$ Protocolo utilizado foi claro, com questões bem definidas e testadas previamente, tendo boa aceitação para a participação dos discentes.

Dada a impossibilidade operacional de se realizar sorteio aleatório dos estudantes e depois encontrá-los para a aplicação do questionário, utilizou-se como alternativa selecionar os estudantes de acordo com o acesso e controlar proporcionalmente com a distribuição por curso, turno e Centro e ano de ingresso. Esta metodologia mostrouse eficiente e os resultados correspondem à distribuição esperada pelo plano amostral. Devido a este procedimento está garantida a representatividade da amostra com relação ao universo de estudo. Com relação ao tamanho da amostra, este foi maior do que o calculado para os parâmetros do estudo, aumentando a precisão dos resultados.

Apesar de muitos estudos abordando o tema da atividade física, ainda existem dificuldades de comparação dos resultados devido a diferenças nas metodologias utilizadas $^{13}$. Nesta pesquisa utilizou-se o IPAQ longo, cuja metodologia já foi validada no Brasil $^{20}$, garantindo a confiabilidade dos resultados. Entretanto, ainda são escassos os estudos representativos com populações de universitários, nacionais e internacionais que tenham utilizado esse instrumento.

Pesquisa realizada com graduandos do curso de Medicina no interior do Estado de São Paulo, em 2003, observou 43,1\% dos estudantes com baixo nível de atividade física $^{21}$. Entre discentes norte-americanos de uma universidade rural, a prevalência deste padrão de atividade física foi de $19,6 \%^{22}$. O resultado observado entre os estudantes da UFPB foi intermediário a estas duas prevalências, mostrando a consistência dos achados. Estas diferenças podem ser atribuídas também a variações das metodologias utilizadas, uma vez que nenhum destes estudos utilizou o IPAQ.

As características associadas com o baixo nível de atividade física que permaneceram no modelo multivariado foram relativas ao perfil institucional do aluno (ano de ingresso, turno que estuda e horas diárias despedidas na universidade), as características econômicas (renda mensal, classe social e com quem reside) e hábitos de vida (fazer ou não dieta).

O menor nível de atividade física dos estudantes com mais tempo de ingresso, quando comparado com os recém ingressados na universidade, observado neste trabalho indica uma tendência de redução 
da atividade física durante este período. Este fenômeno também foi descrito em dois estudos, um canadense e outro brasileiro. No primeiro caso, após o ingresso na Universidade, $31,1 \%$ dos estudantes passaram a ser insuficientemente ativos ${ }^{25}$, e no segundo, em uma Universidade do Estado de Minas Gerais, somente os alunos do curso de educação física, entre todos os demais alunos, mantiveram constante o nível de atividade física durante a graduação ${ }^{26}$.

Com relação ao turno e ao tempo diário na universidade não foram encontrados estudos na literatura que confirmassem estes achados. Pode-se justificar que os alunos dos cursos noturnos e os que passam menos tempo na universidade realizam outras atividades, especialmente trabalho, com pouca exigência de esforço físico laboral, característica peculiar dos jovens de grande capacidade intelectual, como são os universitários. Reforça esta hipótese o fato de que realizar trabalho remunerado e custear com recursos próprios as despesas esteve associado com maiores frequências de baixa atividade física; entretanto, estas variáveis perderam significância estatística e não foram incluídas no modelo multivariado.

Sobre as características econômicas, melhores rendas, pertencer às classes sociais mais altas e morar com a família mostrou-se associado a um comportamento fisicamente menos ativo. Possivelmente facilidades como andar mais de carro, TV a cabo, acesso à Internet e não realizar as próprias atividades domésticas podem contribuir para os baixos níveis de atividade física entre os universitários. Não foram encontrados estudos com população semelhante que confirmem estes achados. Em população adulta, estudo de base populacional realizado em Santa Catarina, no município de Joaçaba, utilizando o IPAQ versão curta, encontrou maior inatividade física entre aqueles de maior renda mensal ${ }^{31}$.

Finalmente, a associação de baixa atividade física com não realizar dieta relaciona dois fatores onde provavelmente o segundo determina o primeiro, ou seja, os estudantes que realizam dieta também estão envol- vidos com programas de atividade física, apresentando este grupo menor prevalência de baixa atividade física. Esta relação dieta mais atividade física é universalmente descrita nos programas para perder peso, muito frequentes entre adolescentes e adultos jovens ${ }^{32-34}$.

Não foram encontradas associações entre sexo e idade com baixo nível de atividade física, apesar de esta relação ser descrita em diversos estudos, como o realizado entre estudantes da área de saúde da Universidade de Brasília, onde foi encontrado que os estudantes do sexo masculino desenvolvem maior nível de atividade física ${ }^{35}$. No nosso caso, a não associação pode ser explicada pela semelhança do perfil etário do universo pesquisado e das atividades exigidas aos discentes na universidade, dependente do curso, mas não do sexo.

O tempo mediano gasto sentado, apesar de ser um bom preditor para comportamentos sedentários, não apresentou diferenças entre os grupos analisados, possivelmente porque as respostas utilizam como escala de tempo unidades de horas e minutos, com maior facilidade de resposta para a primeira. Apesar de a mediana não ser afetada pelos valores extremos, neste caso ela foi influenciada pelas respostas mais frequentes. Não foram encontrados resultados de outros estudos para contrapor estes achados.

Sem associação com baixo nível de atividade física, as prevalências observadas de tabagismo e consumo de bebidas alcoólicas confirmaram, para os estudantes da UFPB, a maior prevalência do consumo de álcool sobre o fumo, observado também em estudos com população semelhante em outras regiões do país. Entretanto, os resultados deste estudo mostram valores até cinco vezes menores que os observados entre estudantes de enfermagem de Ribeirão Preto em $1995^{27}$, entre estudantes de uma Universidade Pública paulista, no ano de $2000^{23}$ ou entre estudantes da área de saúde da Universidade Federal da Amazônia, em $2002^{28}$. Estas diferenças podem ter ocorrido devido a um sub-registro desta informação, 
justificado por não se tratar do objetivo principal da pesquisa.

A prática da atividade física é benéfica à saúde e por isso deve ser incentivada no ambiente universitário. Sabe-se que os estudantes estão continuamente sujeitos a trabalhos, prazos de entrega, longas horas de estudo e situações de exaustão física e mental. Pesquisa com universitários croatas identificou que a realização de programas de esporte nos tempos livres diminui as referências a desconfortos em relação à sua saúde ${ }^{26}$.
Os resultados do presente estudo são consistentes e representativos. Estudar o nível de atividade física de universitários é importante para a descrição de aspectos relacionados à saúde desse grupo e que podem servir para a adoção de estratégias e programas de saúde na universidade como medida preventiva para a adoção de hábitos de vida saudáveis e a melhoria da qualidade de vida na fase adulta e na velhice. A Universidade deve servir para a disseminação de saberes, cultura e valores, dentre os quais as atividades físicas não podem estar excluídas.

\section{Referências}

1. Seclén-Palacin, JA, Jacoby ER. Fatores sociodemográficos y ambientales asociados com la actividad física deportiva em la población urbana del Peru. Rev Panam Salud Publica/Pan Am J Public Health 2003; 4(14): 255-64.

2. Boreham C, Robson PJ, Gallagher AM, Cran GW, Savage M, Murray LJ. Tracking of physical activity, fitness, body composition and diet from adolescence to young adulthood: The Young Hearts Project, Northern Ireland. Int J Behav Nutr Physical Activity 2004 [FALTA INCLUIR VOLUME, NÚMERO E NÚMEROS DE PÁGINAS].

3. Trolle-Lagerros Y, Mucci LA, Kumle M, Braaten T, Tonje Weiderpass E, Chung-Cheng et al. Physical activity as a determinant of mortality in women. Epidemiology 2005; 16(6): 780-875.

4. Pitsavos C, Panagiotakos DB, Lentzas Y, Stefanadis C. Epidemiology of leisure-time physical activity in sociodemographic, lifestyle and psychological characteristic of men and women in Greece: the ATTICA Study. BMC Public Health. BioMed Central 2005. Disponível em http://www.biomedcentral.com [Acessado em julho de 2006].

5. Adams J. Trends in physical activity and inactivity amongst US 14-18 year olds by gender, school grade and race, 1993-2003: evidence from the youth risk behavior survey. BMC Public Health 2006; 6(57): 1-7. Disponível em www.biomedcentral.com/1471-2458/6/57 [Acessado em agosto de 2006].

6. Brasil. Ministério da Saúde. Inquérito domiciliar sobre comportamento de risco e morbidade referida de doenças e agravos não transmissíveis: Brasil, 15 capitais e Distrito Federal, 2002 - 2003. Rio de Janeiro; 2004.

7. Pitanga FJG, Lessa I. Prevalência e fatores associados ao sedentarismo no lazer em adultos. Cad Saúde Pública 2005; 21(3); 870-7.
8. Andrade Filho CW, Malo M. Prevenção de doenças crônicas um investimento vital. Disponível em http:// ops.org. [Acessado em fevereiro de 2006].

9. Ferreira I, Van der Horst K, Wendel-Vos W, Kremers S, Van Lenthe FJ, Brug J. Environmental correlates of physical activity in youth - a review and update. Obesity Reviews 2006; 7 [FALTA INCLUIR NÚMERO E NÚMEROS DE PÁGINAS].

10. Haase A, Phil AS, Sallis JF, Wardle J. Leisure-time physical in university students from 23 countries: associations with health beliefs, risk awareness, and national economic development. Prev Med 2004; 39(1): 182-90.

11. Arrivillaga M, Salazar IC, Correa D. Creencias sobre la salud y su relación com lãs práticas de riesgo o de protección em jóvenes universitários. Colombia Medica 2003; 34(4): 186-95. Disponível em www.biomedcentral. com/1471-2458/6/57 [Acessado em agosto de 2006].

12. Phil AS, Wardle J, Cui W, Bellisle F, Zotti A, Baranyai R et al. Trends in smoking, diet, physical Exercise, and attitudes toward health in European university students from 13 countries, 1990-2000. Prev Med 2002; 35(2): 97 104.

13. Hallal PC, Dumith SC, Bastos JP, Reichert FF, Siqueira FV, Azevedo MR. Evolução da pesquisa epidemiológica em atividade física no Brasil: revisão sistemática. Rev Saúde Pública 2007; 41(3): 453-60.

14. Silva NN. Amostragem Probabilística: um curso introdutório. 2a. ed. São Paulo: Editora da Universidade de São Paulo; 2004.

15. Programa para Análise Epidemiológica de Dados Tabulados - EPIDAT. Direção Geral de Saúde Pública Junta de Galícia-Espanha, Organização Pan-Americana de Saúde, Instituto Superior de Ciências Médicas de Havana-Cuba. Versão 3.1; 2006. 
16. Associação Nacional de Empresas de Pesquisa. Critérios de classificação econômica Brasil, 2000. Disponível em http://www.anep.org.br_[Acessado em fevereiro de 2007].

17. Guideline for Data Processing and Analysis of the International Physical Activity Questionnaire (IPAQ), 2005. Disponível em: http://www.ipaq.ki.se [Acessado em julho de 2006].

18. Guedes DP, Guedes JERP. Manual Prático para Avaliação Física. São Paulo: Manole; 2006.

19. Coutinho W. (Coord.) Consenso latino-americano sobre obesidade - 1998. Disponível em http://www.abeso.org. br [Acessado em agosto de 2006].

20. Pardini R, Matsudo S, Araújo T, Matsudo V, Andrade E, Braggion $\mathrm{G}$ et al. Validação do questionário internacional de nível de atividade física (IPAQ-versão6): estudo piloto em adultos jovens brasileiros. $\mathrm{R}$ Bras Ci $\mathrm{e}$ Mov 2001; 0(3): 45-51.

21. Coelho VG, Loeni FC, Liveratore Júnior RDR, Cordeiro JA, Souza DRS. Perfil lipídico e fatores de risco para doenças cardiovasculares em estudantes de medicina. Arq Bras Cardiol 2005; 85(1): [FALTA INSERIR NÚMEROS DE PÁGINAS].

22. Eisenmann JC, Rates RT, Damori KD. Moderate to vigorous physical activity and weight status in rural university students. J Phys Activ Health 2004; 1: 209-17.

23. Silva LVER, Malbergier A, StempliukVA, Andrade AG. Fatores associados ao consumo de álcool e drogas entre estudantes universitários. Rev Saúde Pública 2006; 40(2): 280-8.

24. Vaez M, Laflamme L. Health behaviors, self-rated health, and quality of life: a study among first-year Swedish university students. J Am Coll Health 2003; 57(4): [FALTA INSERIR NÚMEROS DE PÁGINAS].

25. Bray SR, Born HA. Transition to university and vigorous physical activity: implications for health and psychological well being. J Am Coll Health 2004; 52(4): [FALTA INSERIR NÚMEROS DE PÁGINAS].

26. Silva GSF, Bergamaschine R, Rosa M, Melo C, Miranda R, Bara Filho M. Avaliação do nível de atividade física de estudantes de graduação da áreas saúde/biológica. Rev Bras Med Esporte 2007; 13(1): [FALTA INSERIR NÚMEROS DE PÁGINAS].
27. Moraes SA, Meira L, Freitas ICM. Fatores de risco para doenças crônicas não transmissíveis, entre alunos de enfermagem de Ribeirão Preto - Brasil. Medicina 2000; 33: 312-21.

28. Lucas ACS, Parente RCP, Picanço NS, Conceição DA, Costa KRC, Magalhães IRS et al. Uso de psicotrópicos entre universitários da área de saúde da Universidade Federal do Amazonas, Brasil. Cad Saúde Pública 2006; 22(3): 663-71.

29. Vieira VCR, Priori SE, Ribeiro SMR, Franceschini SCC, Almeida LP. Perfil socioeconômico, nutricional e de saúde de adolescentes recém-ingressos em uma universidade pública brasileira. Rev Nutrição 2002; 3(13): 273-82.

30. Arlis RM.. Cigarette smoking, binge drinking, physical activity, and diet in 138 Asian American and Pacific Islander community college students in Brooklyn, New York. J Comm Health 2006; [FALTA INSERIR VOLUME, NÚMERO E NÚMEROS DE PÁGINAS].

31. Baretta E, Baretta M, Peres KG. Nível de atividade física e fatores associados em adultos no Município de Joaçaba, Santa Catarina, Brasil. Cad Saúde Pública 2007; 23(7): 1595-602.

32. Magalhães VC, Mendonça GAS. Prevalência e fatores associados a sobrepeso e obesidade em adolescentes de 15 - 19 anos, da região Nordeste e Sudeste do Brasil, 1996-1997. Cad Saúde Pública 2003; 19(S1); 129-39.

33. Monteiro RCA, Riether PTA, Burini RC. Efeito de um programa misto de intervenção nutricional e exercício físico sobre a composição corporal e os hábitos alimentares de mulheres obesas em climatério. Rev Nutrição 2004; 17: 478-89.

34. Parente E, Guazzelli I, Ribeiro MM.. Perfil lipídico em crianças obesas: efeitos de dieta hipocalórica e atividade física aeróbica. Arq Bras Endocrinol Metab 2006; 3(50): 499-504.

35. Marcondelli P, Costa THM, SCHMITZ BA. Nível de atividade física e hábitos alimentares de universitários do $3^{\circ}$ ao $5^{\circ}$ semestres da área da saúde. Rev Nutrição 2008; 21(1): 39-47.

Recebido em: 30/04/08 Versão final reapresentada em: 05/02/09 Aprovado em: 18/02/09 